\title{
A novel in vivo model of tau propagation with rapid and progressive neurofibrillary tangle pathology: the pattern of spread is determined by connectivity, not proximity
}

\author{
Zeshan Ahmed · Jane Cooper · Tracey K. Murray • Katya Garn • Emily McNaughton • Hannah Clarke • \\ Samira Parhizkar • Mark A. Ward • Annalisa Cavallini · Samuel Jackson • Suchira Bose $\cdot$ Florence Clavaguera \\ Markus Tolnay • Isabelle Lavenir • Michel Goedert • Michael L. Hutton • Michael J. O’Neill
}

Received: 11 October 2013 / Revised: 28 January 2014 / Accepted: 30 January 2014 / Published online: 16 February 2014

(C) Springer-Verlag Berlin Heidelberg 2014

\begin{abstract}
Intracellular inclusions composed of hyperphosphorylated filamentous tau are a hallmark of Alzheimer's disease, progressive supranuclear palsy, Pick's disease and other sporadic neurodegenerative tauopathies. Recent in vitro and in vivo studies have shown that tau aggregates do not only seed further tau aggregation within neurons, but can also spread to neighbouring cells and functionally connected brain regions. This process is referred to as 'tau propagation' and may explain the stereotypic progression of tau pathology in the brains of Alzheimer's disease patients. Here, we describe a novel in vivo model of tau propagation using human P301S tau transgenic mice infused unilaterally with brain extract containing tau aggregates. Infusion-related neurofibrillary tangle pathology was first observed 2 weeks post-infusion and increased in a stereotypic, time-dependent manner. Contralateral and anterior/posterior spread of tau pathology
\end{abstract}

Electronic supplementary material The online version of this article (doi:10.1007/s00401-014-1254-6) contains supplementary material, which is available to authorized users.

Z. Ahmed · J. Cooper · T. K. Murray · K. Garn ·

E. McNaughton · H. Clarke · S. Parhizkar · M. A. Ward ·

A. Cavallini $\cdot$ S. Jackson $\cdot$ S. Bose $\cdot$ M. L. Hutton .

M. J. O'Neill ( $\square)$

Eli Lilly and Company, Erl Wood Manor, Windlesham,

Surrey GU20 6PH, UK

e-mail: oneill_michael_j@lilly.com

Z. Ahmed

e-mail: ahmedze@lilly.com

F. Clavaguera $\cdot$ M. Tolnay

Department of Neuropathology, Institute of Pathology,

University Hospital, 4031 Basel, Switzerland

I. Lavenir · M. Goedert

MRC Laboratory of Molecular Biology, Cambridge CB2 0QH, UK was also evident in nuclei with strong synaptic connections (efferent and afferent) to the site of infusion, indicating that spread was dependent on synaptic connectivity rather than spatial proximity. This notion was further supported by infusion-related tau pathology in white matter tracts that interconnect these regions. The rapid and robust propagation of tau pathology in this model will be valuable for both basic research and the drug discovery process.

Keywords Tau $\cdot$ Aggregation $\cdot$ Propagation $\cdot$ Prion $\cdot$ Transgenic mouse line $\cdot$ Human P301S tau

\section{Introduction}

Alzheimer's disease (AD) is a neurodegenerative disorder that is characterised by extracellular amyloid plaques and intracellular neurofibrillary tangles (NFTs) [20], with the latter being composed of microtubule-associated protein tau [6]. Tau normally stabilises microtubules and promotes their assembly [15, 48], but in NFTs it is hyperphosphorylated and forms insoluble filamentous aggregates [22]. Abnormal tau accumulation is also characteristic of other neurodegenerative diseases, including progressive supranuclear palsy (PSP), corticobasal degeneration (CBD) and Pick's disease (PiD), which are collectively termed tauopathies [31]. Pathogenic mutations in the tau gene (MAPT) give rise to frontotemporal lobar degeneration (FTLD-Tau), indicating that tau dysfunction is sufficient to cause neurodegeneration and dementia [25, 38, 44]. In AD the severity of NFT pathology correlates with cognitive decline, further supporting the link between tau pathology, functional impairment and neurodegeneration [47]. These studies highlight abnormal tau as a potential therapeutic target and emphasise the need for understanding the molecular 
Table 1 Treatment groups, time points and sample sizes

\begin{tabular}{lllll}
\hline Time (PI) & Age (months) & \multicolumn{3}{l}{ Treatment groups $(n)$} \\
\cline { 3 - 5 } & & ${ }^{\mathrm{P} 301 \mathrm{~S}} \mathrm{BE}$ & $\mathrm{PBS}$ & ${ }^{\mathrm{WT}} \mathrm{BE}$ \\
\hline 1 day & 2 & 5 & 5 & - \\
2 weeks & 2.5 & 5 & - & - \\
1 month & 3 & 5 & 5 & - \\
2 months & 4 & 5 & - & - \\
2.5 months & 4.5 & $10^{\mathrm{a}}$ & 5 & 8 \\
\hline
\end{tabular}

Mice were unilaterally infused with P301S brain extract $\left({ }^{\mathrm{P} 301 \mathrm{~S}} \mathrm{BE}\right)$, sterile phosphate-buffered saline (PBS) or wild-type brain extract $\left({ }^{\mathrm{WT}} \mathrm{BE}\right)$, and were aged to pre-defined time points

$P I$ post-infusion

a 5 mice used for biochemistry

mechanism(s) of tau-associated neurodegeneration, which will be critical for developing novel therapeutic strategies.

NFTs appear in a progressive and stereotypical fashion, which enabled Braak and Braak to stage their severity and distribution [5]: stages I-II are thought to represent prodromal AD, with NFTs largely confined to the entorhinal cortex; stages III-IV are associated with mild cognitive impairment and hippocampal involvement, whereas stages V-VI represent clinically apparent AD and NFTs extending into the neocortex [5]. Only recently, this apparent induction and spread of tau pathology has been demonstrated experimentally.

Clavaguera et al. [9] showed that abnormally phosphorylated, filamentous tau derived from the brains of human P301S tau transgenic mice was sufficient to induce the formation of silver-positive tau inclusions in ALZ17 mice that overexpress wild-type human tau, but do not develop tau inclusions. They also showed that tau pathology developed progressively and spread from the sites of infusion to neighbouring brain regions. This was consistent with in vitro data showing that tau aggregates can be internalised by cultured cells, resulting in the fibrillisation of expressed tau [17]. These studies have suggested that tau aggregates, like extracellular amyloids [19, 43], can recruit monomeric tau into fibrillar aggregates that spread to neighbouring brain regions through an as yet unknown mechanism. This process is referred to as 'tau propagation' and has been proposed to explain the stereotypic progression of NFT pathology $[16,42]$. There is also a growing body of evidence to suggest that tau pathology may spread via synaptically connected networks. Using bigenic mice, which overexpressed human P301L tau apparently specifically in layer II neurons of the entorhinal cortex, tau pathology was shown to spread from this region to hippocampal neurons in a progressive fashion, despite there being no detectable transgene expression in the hippocampus $[12,33]$. Although these experimental models help to elucidate the mechanism(s) of tau propagation, there are limitations from the perspective of developing novel tau-based therapies. The ALZ17 propagation model takes 15 months before robust tau pathology is evident, with no apparent neurodegeneration [9]. The region-specific expression models take 18 months to develop robust propagation-related pathology $[12,33]$ and the possibility of low human mutant tau expression being responsible for the apparent synaptic spread cannot be ruled out [42].

In the present study, we used the human P301S tau transgenic line to develop an accelerated model of tau propagation and to study the relationship between tau spread and synaptic connectivity. The P301S tau transgenic model overexpresses the $0 \mathrm{~N} 4 \mathrm{R}$ isoform of human tau containing the P301S mutation under the murine thyl promoter [1]. Homozygous mice from this line develop a severe paraparesis, robust neuronal tau pathology (hyperphosphorylated and fibrillar) and extensive neurodegeneration by 5-6 months of age. Although some tau pathology has been reported in the forebrain of P301S tau mice, the brunt of pathology is present in brainstem and spinal cord [1].

\section{Materials and methods}

\section{Transgenic mice}

The generation of homozygous P301S tau transgenic mice has been reported previously [1]. Mice (line 2541) were bred on a mixed C57BL/6JxCBA/ca background. Wildtype (WT) mice on a C57/BL/6J background were obtained from Charles River (Margate, UK).

Preparation of brain extracts and infusion procedure

All procedures were performed in accordance with the approved Eli Lilly Ethical Review board and UK Home Office guidelines. Brain extracts were prepared from endstage (5-5.5 months) P301S tau mice and age-matched WT controls, as previously described [9]. In brief, mice were killed via dislocation of the neck and decapitation; brain stems were rapidly dissected on wet ice and snap frozen on dry ice. Frozen brainstems from five mice were combined and homogenised at $10 \%(\mathrm{w} / \mathrm{v})$ in sterile phosphate-buffered saline (PBS), briefly sonicated (Branson 450, output $2,5 \times 0.9 \mathrm{~s}$ ) and centrifuged at $3,000 \mathrm{~g}$ at $4{ }^{\circ} \mathrm{C}$ for $5 \mathrm{~min}$. The supernatant was stored at $-80{ }^{\circ} \mathrm{C}$ until use; see supplementary material for concentration of protein and tau in brain extracts.

Female P301S tau mice were randomly assigned to three groups and five time points, as summarised in Table 1 . Two-month-old P301S tau mice were anaesthetised with isoflurane $\left(2-3 \%\right.$ in $\left.\mathrm{O}_{2}, 2 \mathrm{l} / \mathrm{min}\right)$ following sedation with 
Table 2 Antibodies used for immunohistochemistry

\begin{tabular}{lllll}
\hline Antibody & Epitope & Species & Dilution & Source \\
\hline PG5 & pSer409 & Mouse & $1: 8,000$ & Kind gift from Peter Davies \\
MC1 & Conformation dependent & Mouse & $1: 32,000$ & Kind gift from Peter Davies \\
AT8 & pSer202 and pThr205 & Mouse & $1: 2,000$ & Innogenetics \\
TG3 & pThr231 and conformation dependent & Mouse & $1: 3,000$ & Kind gift from Peter Davies \\
IBA-1 & Ionised calcium binding adaptor 1 & Rabbit & $1: 6,000$ & Wako Chemicals \\
\hline
\end{tabular}

medetomidine (Domitor@ $1 \mathrm{mg} / \mathrm{kg}$ s.c.). Once deeply anaesthetised, they were placed in a stereotaxic frame and a heating blanket with a rectal probe used to maintain body temperature. Maintenance anaesthesia was given at $1 \%$ isoflurane in $0.5 \mathrm{l} / \mathrm{min} \mathrm{O}_{2}$. Once the skull was exposed and cleaned, a hole was drilled over the appropriate coordinates and brain extract from P301S tau mice $\left({ }^{\mathrm{P} 301 \mathrm{~S}} \mathrm{BE}\right)$ or WT mice ( ${ }^{\mathrm{WT}} \mathrm{BE}$ ) was infused; a third group was infused with sterile PBS. Each mouse received two infusions into the left hemisphere (Supplementary Fig. 1e). The hippocampus (bregma: AP -2.50, LM +2.00; dural surface: DV $-1.80)$ and then the overlying cerebral cortex/white matter (bregma: AP - 2.50, LM +2.00; dural surface: DV -0.80) were unilaterally infused via a 28-gauge cannula (Plastics One, VA, USA) attached to an infusion pump (CMA100, CMA, Solna, Sweden) and fluorinated ethylene propylene tubing. Each infusion was $2.5 \mu \mathrm{l}$ at a flow rate of $1.25 \mu \mathrm{l} /$ min with the cannula remaining in place for a further 3 min before withdrawal. Following infusion the skin was sutured, sedation was reversed with atipamezole (Antisedan $(5 \mathrm{mg} / \mathrm{kg}$ s.c.) and the mice were allowed to recover with postoperative analgesia (Rimadyl@ $5 \mathrm{mg} / \mathrm{kg}$ s.c.) in a temperature-controlled environment prior to returning to their home cages. Mice were weighed and checked daily for 2 weeks following surgery, with access to rehydration sachets and food and water ad libitum.

Histology and immunohistochemistry

At specified time points, mice were terminally anaesthetised and transcardially perfused with $30 \mathrm{ml}$ saline, followed by $30 \mathrm{ml} 10 \%$ buffered formalin. Brains were removed and post-fixed in $10 \%$ buffered formalin overnight, before being coronally dissected into three segments using an adult mouse brain matrix (RBM-2000C; ASI Instruments, MI, USA). These segments were processed using the Tissue TEK $^{\circledR}$ VIP processor (GMI Inc, MN, USA) and embedded in paraffin wax. From the middle segment, $7 \mu \mathrm{m}$ serial sections (from bregma 1.00 to -4.50 ) were cut using rotary microtomes (HM 200 and HM 355; Thermo Scientific, Microm, Germany) and mounted on glass slides (three sections per slide). Immunohistochemistry was performed using the primary antibodies listed in Table 2 and is described in supplementary materials. Gallyas silver [18] and thioflavin-S [40] staining were performed as previously described, with the former being counterstained with methyl green (Dako, Cambridgeshire, UK) and the latter with DAPI (Vector Laboratories, CA, USA).

Neuropathological characterisation

Stained sections (3 per slide) were digitised using the Scanscope XT slide scanner (Aperio, CA, USA) at $20 \times$ magnification. Imagescope software (version 11.1.2.760; Aperio, CA, USA) was used to view, delineate and analyse digitised tissue sections. Supplementary Fig. 1 shows the regions of interest (ROIs) that were delineated and their relative coronal level. To analyse tau pathology, the number of PG-5- or Gallyas-positive neurons was manually counted within a delineated ROI and expressed as density $\left(\mathrm{mm}^{2}\right)$. For some ROIs, PG-5 immunoreactivity was quantified using the positive pixel algorithm (Imagescope, version 11.1.2.760; Aperio, CA, USA) and expressed as the percentage of area burden; a similar approach was used to quantify Iba-1-positive microglia in dentate gyrus, layer CA3 and layer CA1. In white matter (fornix and internal capsule), tau pathology was measured using a four-tier semi-quantitative scoring scheme ranging from absent (0) to severe (3). These analyses were performed blind: (1) on the infused and contralateral sides of each ROI and brain; (2) in triplicate on serial tissue sections (mean used for statistical analysis).

Biochemical analysis

A subset of ${ }^{\mathrm{P} 301 \mathrm{~S}} \mathrm{BE}$-infused mice (2.5 months post-infusion) were killed by cervical dislocation followed by rapid removal of the brain and immediate dissection (as shown in Supplementary Figure 3); individual brain segments were snap frozen and stored at $-80{ }^{\circ} \mathrm{C}$. Tissue samples were homogenised in ice-cold homogenisation buffer (supplementary materials) using $15 \times 1 \mathrm{~s}$ sonication pulses. Following homogenisation samples were centrifuged at $13,000 \mathrm{~g}$ for $10 \mathrm{~min}$ at $4{ }^{\circ} \mathrm{C}$ to generate the low-speed fractions (LS). Protein-normalised LS fraction was further centrifuged at $100,000 \mathrm{~g}$ for $30 \mathrm{~min}$ at $4{ }^{\circ} \mathrm{C}$ to separate the soluble ( $\mathrm{S} 1$, supernatant) and insoluble ( $\mathrm{P} 1$, pellet) fraction. The $\mathrm{P} 1$ pellets were washed and probe sonicated in 
$50 \mathrm{mM}$ Tris pH 7.4. Optimised AlphaScreen assays were performed using tau-specific antibodies as per manufacturer's instructions (see supplementary materials) and Western blotting was preformed as previously described [7].

\section{Statistical analysis}

Quantitative data were analysed using Student's $t$ test for pairwise comparisons and one-way ANOVA, followed by either Bonferroni's or Dunnett's post hoc tests for groupwise comparisons. Semi-quantitative data were analysed using Mann-Whitney test for pairwise comparisons and Kruskal-Wallis test, followed by Dunn's post hoc test for groupwise comparisons. Correlation analyses were performed using Spearman's rank correlation coefficient. GraphPad Prism 5 (version 5.04; GraphPad software Inc., CA, USA) was used to perform statistical tests and to produce graphs, which display mean values \pm SEM. Statistical significance was set at $P<0.05$, with a trend towards significance defined as $P=0.05-0.08$.

\section{Results}

Induction of severe tau pathology in the hippocampus of P301S mice infused with ${ }^{\mathrm{P} 301 \mathrm{~S}} \mathrm{BE}$

Examination at the infusion coordinates 2.5 months postinfusion revealed striking PG-5-positive tau pathology in the hippocampus of ${ }^{\mathrm{P} 301 \mathrm{~S}} \mathrm{BE}$ mice, whereas such pathology was only sparse in age-matched controls (infused with either ${ }^{\mathrm{WT}} \mathrm{BE}$ or PBS) (Fig. 1a). Tau pathology in the hippocampus of ${ }^{\mathrm{P} 301 \mathrm{~S}} \mathrm{BE}$ mice was severe on the infused side, but also spread to the contralateral side, consistent with propagation. Tau pathology was limited to neurons and glial tau pathology was not observed. Neurons in all hippocampal subregions were affected, but tau pathology was particularly severe in the dentate gyrus and the proximal two-thirds of layers CA3 and CA1. Quantification of PG-5-positive neurons in the hippocampus (Supplementary Fig. 1) of ${ }^{\mathrm{P} 301 \mathrm{~S}} \mathrm{BE}-$-, ${ }^{\mathrm{WT}} \mathrm{BE}-$ and PBS-infused mice 2.5 months post-infusion confirmed these observations (Fig. 1b). The density of PG-5-positive inclusions was significantly greater in the infused and contralateral hippocampi of ${ }^{\mathrm{P} 301 \mathrm{~S}} \mathrm{BE}$ mice than in ${ }^{\mathrm{WT}} \mathrm{BE}$ or PBS controls. In mice infused with ${ }^{\mathrm{P} 301 \mathrm{~S}} \mathrm{BE}$, the infused hippocampus had significantly more inclusions than the contralateral hippocampus, whereas no such differences were detected in the ${ }^{\mathrm{WT}} \mathrm{BE}$ or PBS groups. A similar analysis of hippocampal subregions (Supplementary Fig. 2) and biochemical analysis of tau in the hippocampus of ${ }^{\mathrm{P} 301 \mathrm{~S}} \mathrm{BE}$-infused mice (Supplementary Fig. 3) was consistent with the above results. Tau-positive neuronal inclusions in ${ }^{\mathrm{P} 301 \mathrm{~S}} \mathrm{BE}$-infused
Fig. 1 Characterisation of ${ }^{\mathrm{P} 301 \mathrm{~S}} \mathrm{BE}$-induced hippocampal tau pathology. a At 2.5 months post-infusion, PG-5 immunohistochemistry identified severe neuronal tau pathology in the hippocampus (dentate gyrus, CA3 and CA1) of mice infused with ${ }^{\mathrm{P} 301 \mathrm{~S}} \mathrm{BE}$, whereas age-matched controls infused with either ${ }^{\mathrm{WT}} \mathrm{BE}$ or PBS showed only sparse tau pathology. Neuronal tau pathology in ${ }^{\mathrm{P} 301 \mathrm{~S}} \mathrm{BE}$ mice was: (1) accompanied by neuropil threads, (2) more severe on the infused than the contralateral side and (3) Gallyas silver positive. b Quantification of PG-5-positive neurons in the infused (solid shapes) and contralateral (empty shapes) hippocampi showed significant $(P<0.05)$ differences between ${ }^{\mathrm{P} 301 \mathrm{~S}} \mathrm{BE}-($ red $)$ and ${ }^{\mathrm{WT}} \mathrm{BE}$ - (blue) or PBS- (green) infused mice; in addition, the infused and contralateral hippocampi of ${ }^{\mathrm{P} 301 \mathrm{~S}} \mathrm{BE}$ mice showed a significant difference. $\mathbf{c}$ At this time-point, PG-5-positive tau pathology could also be detected by anti-tau antibodies AT8 (recognising tau phosphorylated at Ser202/Thr205), MC1 (conformation-dependent tau epitope) and TG3 (phosphorylation of tau at Thr231 and conformation-dependent), with staining for the latter being more restricted. Confocal microscopy showed that a small subset of neuronal inclusions was Thioflavin-S-positive with limited neuropil thread staining (white arrows); such inclusions were absent in age-matched control mice (data not shown). Statistics: Groupwise comparison, one-way ANOVA with Bonferroni's post hoc test; ****, $P<0.0001$. $B E$ brain extract, $P B S$ phosphate-buffered saline, $W T$ wild type. Scale bars: a, $200 \mu \mathrm{m} ; \mathbf{b}, 50 \mu \mathrm{m}$

mice had a range of distinct cytoplasmic morphologies (globular, perinuclear or large spherical) and were often associated with neuropil threads. They were also strongly Gallyas positive (Fig. 1a), indicating that tau was in a fibrillar conformation reminiscent of NFTs [4]; additional staining characteristics are shown in Fig. 1c. In contrast, taupositive inclusions in age-matched controls (infused with either ${ }^{\mathrm{WT}} \mathrm{BE}$ or PBS) were mainly diffuse, associated with limited neuropil threads and only weakly Gallyas positive (data not shown); these features are consistent with pretangles [4].

Progressive and stereotypical accumulation of tau inclusions in the hippocampus of ${ }^{\mathrm{P} 301 \mathrm{~S}} \mathrm{BE}$ mice

The temporal profile of ${ }^{\mathrm{P} 301 \mathrm{~S}} \mathrm{BE}$-induced tau propagation was examined in the ipsilateral and contralateral hippocampi of ${ }^{\mathrm{P} 3015} \mathrm{BE}$ mice. Qualitative observations are shown in Fig. 2a, whereas quantitative changes are summarised in Fig. $2 b$, c. 1 day post-infusion: No PG-5 immunoreactivity or Gallyas staining was observed, but disruption of layer CA1 and the dentate gyrus was visible (asterisk in Fig. 2a); these changes were found in both ${ }^{\mathrm{P} 301 \mathrm{~S}} \mathrm{BE}$ and control mice. 2 weeks postinfusion: Numerous PG-5-positive neuronal inclusions were detected in the infused hippocampus, with a small proportion being weakly Gallyas positive. Neuropil thread and contralateral tau pathology was limited. 1 month postinfusion: Compared to the 2 week time point, tau pathology was more severe in the infused hippocampus; it was also present (though milder) in the contralateral hippocampus. On the infused side, PG-5-positive inclusions occupied more of the cell soma and were accompanied by neuropil 


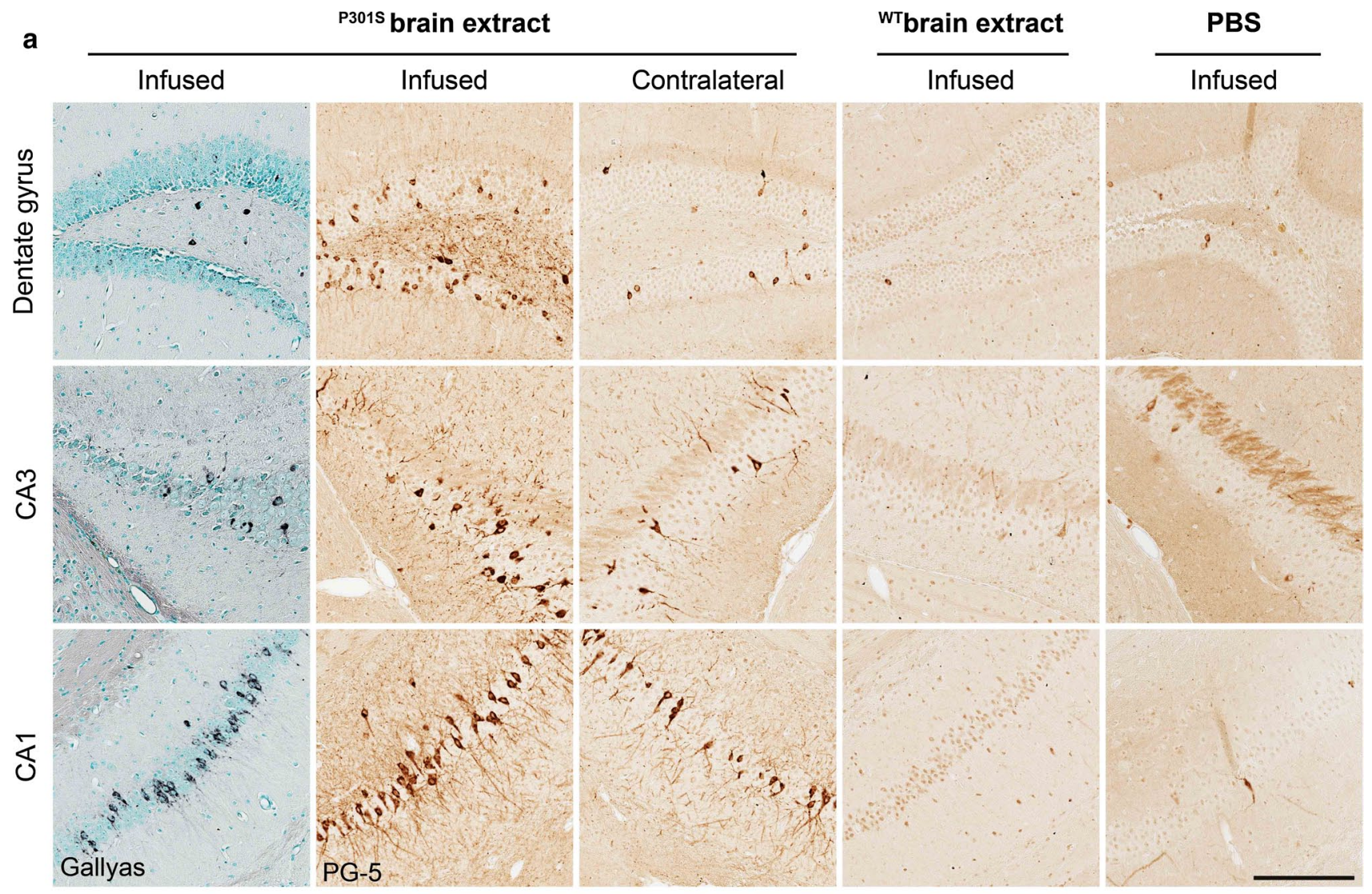

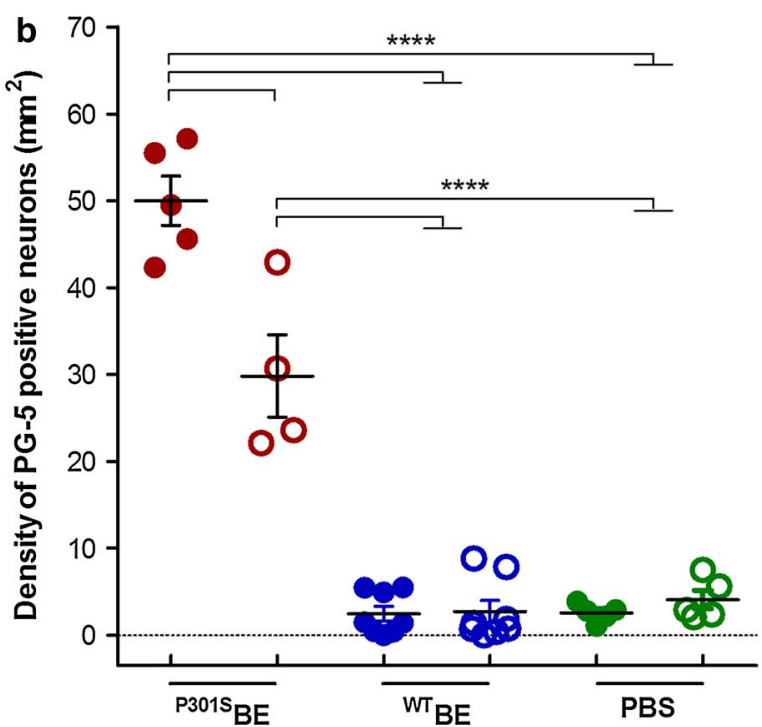

threads. A greater proportion of neuronal inclusions were Gallyas positive. The contralateral hippocampus was similar to infused side at 2 weeks post-infusion, with the exception that the dentate gyrus was relatively spared. 2 months postinfusion: There was no substantial increase in the number of tau inclusions. Most inclusions on the infused side were intensely PG-5 positive, occupied the whole cell soma and were associated with extensive neuropil threads. Similar
C

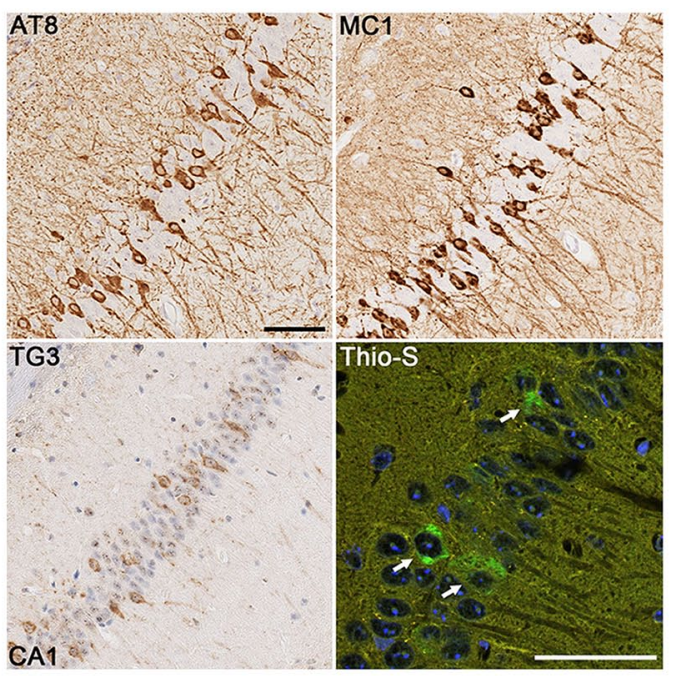

changes were also found on the contralateral side, albeit in milder form, and the dentate gyrus was now also affected. 2.5 months post-infusion: Tau pathology was most severe at this stage (Fig. 1). Most PG-5-positive inclusions were also Gallyas positive, suggesting a predominance of NFTs. Consistent with these qualitative observations, quantification of PG-5-positive neurons in the infused and contralateral hippocampi of ${ }^{\mathrm{P} 301 \mathrm{~S}} \mathrm{BE}$ mice showed a time-dependent increase 
a

Contralateral

Hippocampus

Infused

CA1

${ }^{\text {P301SBE }}$

\section{CA1}

PG-5

PG-5

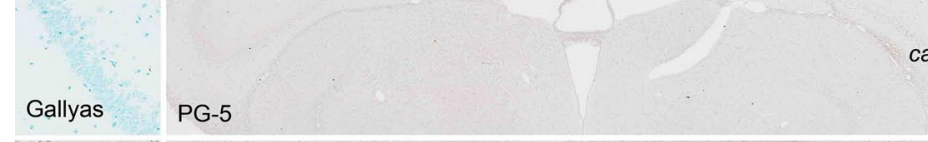

Gallyas PG- ca3

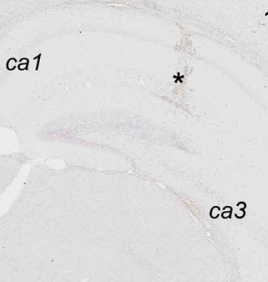

weeks
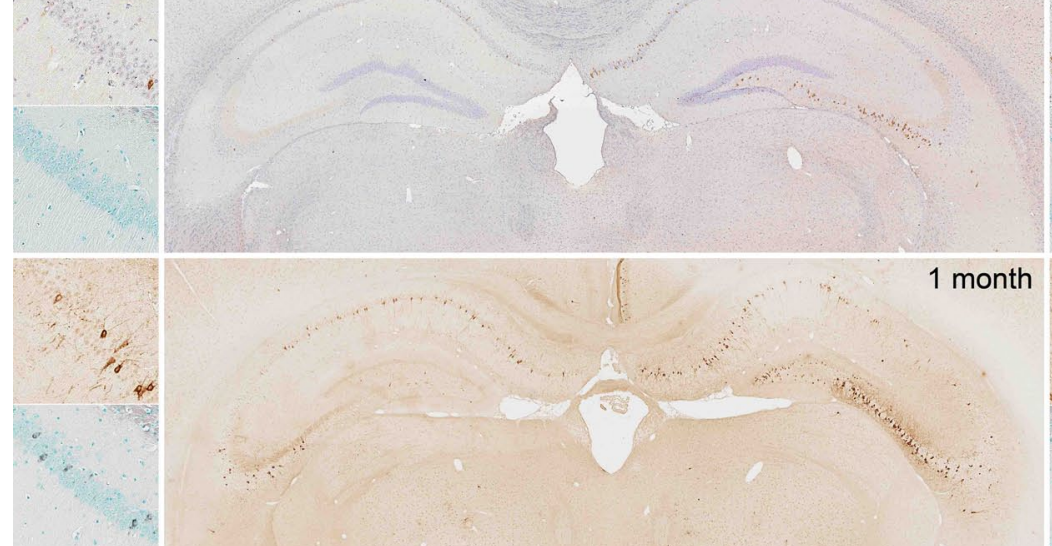

2 months
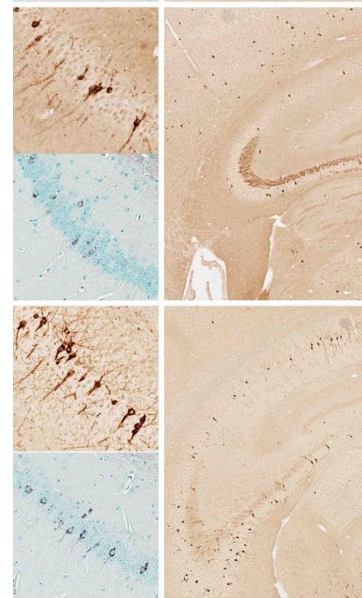

${ }^{\text {WT} B E-i n f u s e d}$

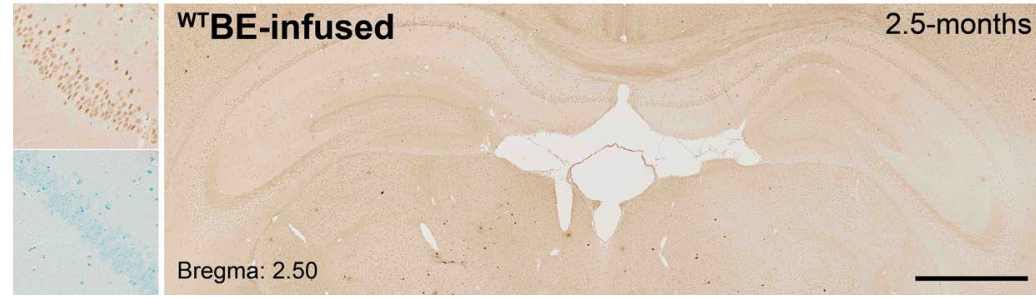

$\therefore$ CA1

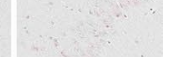


4 Fig. 2 Time-dependent progression of ${ }^{\mathrm{P} 301 \mathrm{~S}} \mathrm{BE}$-induced hippocampal tau pathology. a Representative images showing PG-5-positive tau pathology in the hippocampus (bregma -2.50) of human P301S tau transgenic mice 1 day, 2 weeks, 1 month, 2 months and 2.5 months after the infusion with ${ }^{\mathrm{P} 301 \mathrm{~S}} \mathrm{BE}$, compared to P301S tau mice 2.5 months after the infusion with ${ }^{\mathrm{WT}} \mathrm{BE} / \mathrm{PBS}$. Insets show PG-5 immunohistochemistry and Gallyas silver staining in the CA1 region (performed on adjacent serial sections); those on the right show the infused side and those on the left the contralateral side. Tau pathology was absent at 1 day post-infusion, but mild infusion-related tissue damage was visible on the infused side (indicated by asterisk). From 2 weeks post-infusion, PG-5-positive tau pathology increased in a time-dependent manner on the infused side; neuronal inclusions became more strongly PG-5 positive, were associated with neuropil threads and became increasingly Gallyas silver positive. Similar pathological changes occurred on the contralateral side 1 month post-infusion, where the pathology was comparatively less severe. In contrast, tau pathology was only sparse in the infused and contralateral hippocampus of ${ }^{\mathrm{WT}} \mathrm{BE}$ mice, even 2.5 months post-infusion. b The density of PG-5-positive neurons in the hippocampus showed an age-associated increase on the infused and contralateral sides. From 2 weeks post-infusion, the density of PG-5-positive neurons was significantly $(P<0.05)$ increased in the infused hippocampus of ${ }^{\mathrm{P} 301 \mathrm{~S}} \mathrm{BE}$ mice when compared with ${ }^{\mathrm{WT}} \mathrm{BE} / \mathrm{PBS}$-infused mice 2.5 months postinfusion; similar results were obtained on the contralateral side, but only at 2 months post-infusion. c To investigate the relative proportion of PG-5-positive, silver-negative tau inclusions (pre-tangles) versus PG-5-positive and silver-positive inclusions (NFTs), the number of Gallyas-positive neurons in the CA1 region was expressed as the percentage of the number of PG-5-positive neurons quantified in the same region, but on an adjacent serial section. At 2 weeks postinfusion, the majority of tau inclusions in ${ }^{\mathrm{P} 301 \mathrm{~S}} \mathrm{BE}$-infused mice were pre-tangles, whereas by 2.5 months both the infused and contralateral hippocampi contained predominantly NFTs. In contrast, pre-tangles predominated in PBS-infused mice, even 2.5 months post-infusion. Comparison of ${ }^{\mathrm{P} 301 \mathrm{~S}} \mathrm{BE}$ and PBS groups at 2.5 months post-infusion showed a significant increase in NFTs in the former. Statistics: Groupwise comparison, one-way ANOVA with Dunnett's post hoc test (b) and one-way ANOVA with Bonferroni's post hoc test (c); pairwise comparison, Student's $t$ test (c). BE brain extract, WT wild type, $d$ day, $w$ weeks, $m$ month; * $P<0.05$; **, $P<0.01$; ***, $P<0.001$; ****, $P<0.0001$. Scale bars: a, $1 \mathrm{~mm}$; insets, $100 \mu \mathrm{m}$

Anatomical spread of ${ }^{\mathrm{P} 301 \mathrm{~S}} \mathrm{BE}$-associated tau pathology is related to connectivity, not proximity

The hippocampal formation is made up of the dentate gyrus, layers CA4, CA3, CA2 and CA1, subiculum, presubiculum, parasubiculum and entorhinal cortex and represents a synaptic network that allows the predominately unidirectional passage of information [2]. To test the hypothesis that tau spread is related to synaptic connectivity $[9,12,26,33]$, tau pathology was analysed in brain nuclei with and without strong synaptic connections to the hippocampus. See $[2,36]$ for details on the connectivity of nuclei selected for analysis. The results of this analysis are summarised in Table 3 and selected examples are shown in Fig. 3; analysis data for individual regions are shown in supplementary materials.
Spread of tau pathology within the hippocampal formation

The subiculum is one of the major sources of efferent projections from the hippocampal formation and is heavily innervated by layer CA1 and the entorhinal cortex. Examination of the dorsal subiculum (Bregma -2.50) showed strong evidence of propagation in ${ }^{\mathrm{P} 301 \mathrm{~S}} \mathrm{BE}$ mice, whereas control mice showed only sparse pathology (Fig. 3b). Evidence of tau pathology was observed even at 2 weeks post-infusion, suggesting that it was an early feature (Supplementary Fig. 4), whereas spread to the contralateral side first appeared at 2 months post-infusion (data not shown). Similar to the subiculum, the pre- and para-subiculum (Fig. 3c) also showed evidence of tau propagation, despite being posterior to the infusion site (Bregma -4.50). These regions receive inputs from the subiculum.

Spread of tau pathology to extrahippocampal nuclei

Tau pathology was observed in many nuclei outside the hippocampal formation. Some, like the retrosplenial cortex (RSC; Fig. 3a), were severely affected and showed pathology as early as 2 weeks post-infusion (Supplementary Fig. 4), which increased in a time-dependent manner. Like the subiculum, the RSC is relatively close to the site of infusion (Bregma -2.50) and receives strong synaptic input from the hippocampal (CA1 and subiculum) and para-hippocampal (pre- and post-subiculum) regions. The nucleus accumbens (bregma 1.00) and the anterior thalamic nuclear complex (bregma -1.00) are also synaptically connected to the hippocampal region. The former receives strong and unidirectional synaptic input from the subiculum, whereas the anterodorsal (ADthalamus) and anteroventral (AVthalamus) nuclei of the latter are reciprocally interconnected with the pre-subiculum. Despite being located anteriorly to the infusion site, these nuclei also showed a pattern of pathology that was consistent with tau propagation (Fig. 3g-i). Evidence of propagation was not as obvious as that seen in the RSC, with only the infused side showing propagation to the nucleus accumbens (Fig. 3i) and ADthalamus (Fig. 3g) of ${ }^{\mathrm{P} 301 \mathrm{~S}} \mathrm{BE}$ mice 2.5 months post-infusion.

\section{Bilateral spread of tau pathology}

The lateral septal nucleus (LSN) receives unidirectional, bilateral projections from the hippocampal formation, specifically CA3, CA1 and subiculum, through the fimbria-fornix; this is a prominent connection, as all CA3 and CA1 neurons project to the LSN. Like other synaptically connected regions showing evidence of propagation, the LSN also contained tau pathology despite being anterior (bregma 0.00) to the infusion site. In contrast to regions where the infused side 

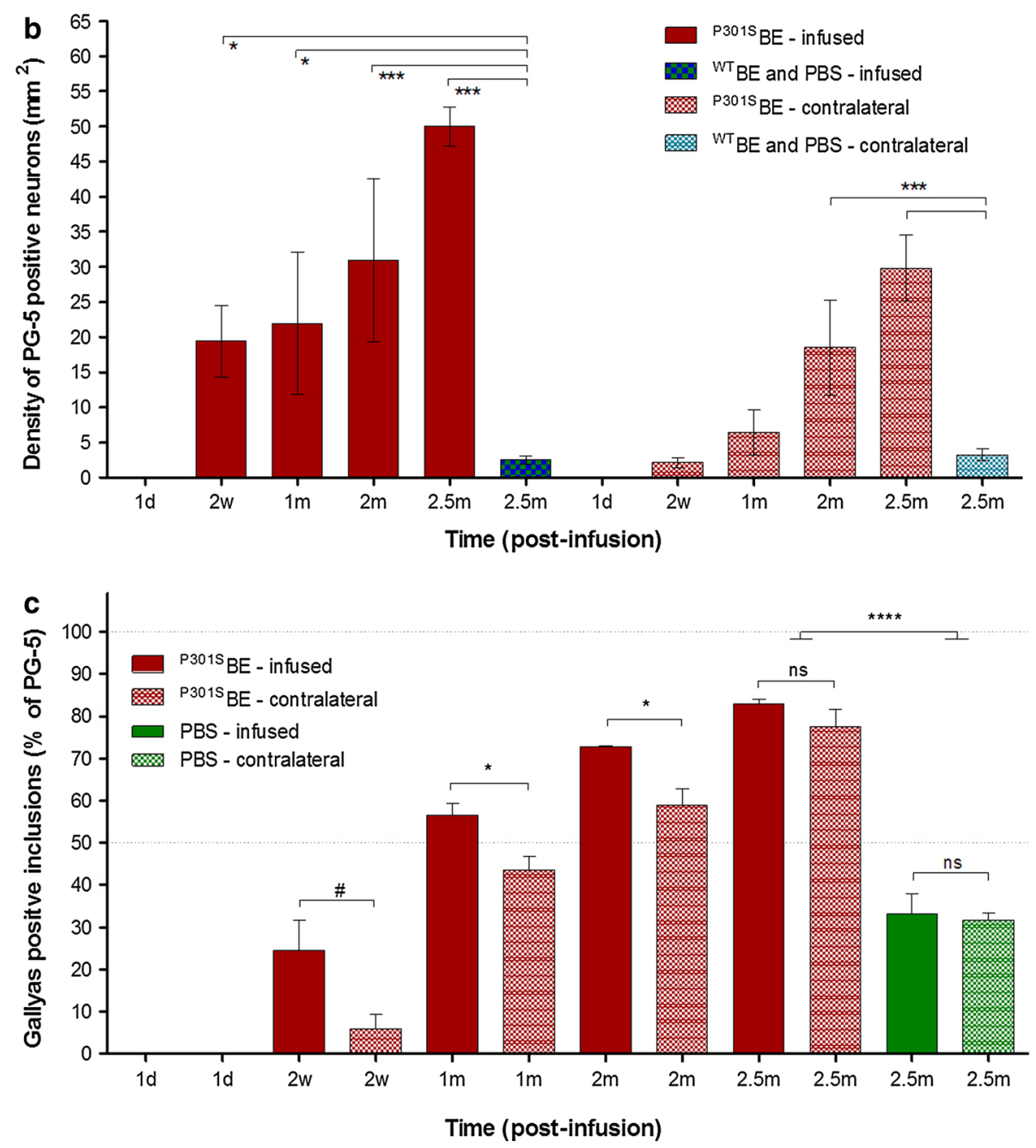

Fig. 2 continued

was more severely affected than the contralateral side, the severity of pathology in the LSN was similar on both sides (Fig. 3f, n). A small subset of ${ }^{\mathrm{P} 301 \mathrm{~S}} \mathrm{BE}$ mice showed mild tau pathology in the LSN at 2 weeks post-infusion, but only on the contralateral side, whereas more substantial pathology was observed on both sides at 1 month post-infusion (data not shown). What distinguishes the LSN from other propagation-associated nuclei is that the projection from layer CA3 is bilateral, which may explain the tau spread to both sides.

Anterograde and retrograde spread of tau pathology

The medial and lateral mammillary nuclei (mlMN) receive strong unidirectional, bilateral inputs from the subiculum and para-hippocampus through the fimbria-fornix. As these connections are not reciprocal, return projections from the mammillary body to the subiculum (and the dentate gyrus) originate from the supramammillary nucleus (suMN). Despite being approximately $5 \mathrm{~mm}$ from the site of infusion, both regions showed statistically significant evidence of tau pathology (Fig. 3d, e), which was particularly prominent in the mlMN (Fig. 3o). Spread from the hippocampal formation to the mlMN is an example of anterograde spread, whereas that to the suMN constitutes retrograde spread. Consistent with the bilateral arrangement of these connections, the severity of tau pathology on the infused and contralateral sides of ${ }^{\mathrm{P} 301 \mathrm{~S}} \mathrm{BE}$ mice was not significantly different. Furthermore, evidence of propagation was observed as early as 2 weeks post-infusion (Supplementary Fig. 5), suggesting that the anterograde and retrograde spread of tau pathology occurred relatively early.

Nuclei with no evidence of propagation-associated tau pathology

The lateral geniculate nucleus (LGN; Bregma -2.50) receives inputs from the retina and projects to the visual cortex via the internal capsule. Although it is not directly 
Table 3 Tau propagation to brain regions distal to the infusion site

\begin{tabular}{|c|c|c|c|c|c|}
\hline Region of interest & $\begin{array}{l}\text { Relationship } \\
\text { to infusion site }\end{array}$ & $\begin{array}{l}\text { Bregma } \\
(\mathrm{A} / \mathrm{P})\end{array}$ & $\begin{array}{l}\text { Direct connection to } \\
\text { hippocampal formation? }\end{array}$ & $\begin{array}{l}\text { Evidence of tau propagation } \\
\text { in }{ }^{\mathrm{P} 01 \mathrm{~S}} \mathrm{BE} \text { mice? }\end{array}$ & Fig. \\
\hline Para-hippocampal region & Posterior & -4.50 & Yes & Yes & $3 \mathrm{c}$ \\
\hline Dentate gyrus (posterior) & Posterior & -4.00 & Yes & Yes & - \\
\hline Retrosplenial cortex & Dorsal & -2.50 & Yes & Yes & $3 \mathrm{a}, 3 \mathrm{~m}$ \\
\hline Dorsolateral cortex & Dorsal & -2.50 & No & No & - \\
\hline Dorsal subiculum & Medial & -2.50 & Yes & Yes & $3 b$ \\
\hline Hippocampus region & Target & -2.50 & Yes & Yes & $1 b$ \\
\hline Dentate gyrus & Target & -2.50 & Yes & Yes & Sup. 2 \\
\hline CA3 & Target & -2.50 & Yes & Yes & Sup. 2 \\
\hline CA1 & Target & -2.50 & Yes & Yes & Sup. 2 \\
\hline \multicolumn{2}{|c|}{ Medial-lateral mammillary nucleusVentral } & -2.50 & Yes & Yes & $3 \mathrm{~d}, 3 \mathrm{o}$ \\
\hline Supramammillary nucleus & Ventral & -2.50 & Yes & Yes & $3 \mathrm{e}$ \\
\hline Ventrolateral cortex & Ventral & -2.50 & Yes & Yes & - \\
\hline Lateral geniculate nucleus & Ventral & -2.50 & No & No & $3 \mathrm{j}$ \\
\hline Anterioventral thalamus & Anterior & -1.00 & Yes & Yes & $3 \mathrm{~h}$ \\
\hline Dentate gyrus (anterior) & Anterior & -1.00 & Yes & Yes & - \\
\hline CA3 (anterior) & Anterior & -1.00 & Yes & Yes & - \\
\hline Anteriodorsal thalamus & Anterior & -1.00 & Yes & Yes & $3 g$ \\
\hline Globus pallidus & Anterior & -1.00 & No & No & $3 \mathrm{k}$ \\
\hline Ventrolateral thalamus & Anterior & -1.00 & No & No & - \\
\hline Lateral septal nucleus (dorsal) & Anterior & 0.00 & Yes & Yes & $3 f, 3 n$ \\
\hline Nucleus accumbens & Anterior & 1.00 & Yes & Yes & $3 \mathrm{i}$ \\
\hline Caudate/putamen & Anterior & 1.00 & No & No & 31 \\
\hline
\end{tabular}

Evidence of tau propagation was analysed in various brains regions of ${ }^{\mathrm{P} 301 \mathrm{~S}}$ BE-infused mice at 2.5 months post-infusion (see Supplementary materials for analysis data). Tau propagation was defined as a region showing significantly more tau pathology in ${ }^{\mathrm{P} 301 \mathrm{~S}} \mathrm{BE}$ mice compared to PBS controls (highlighted in italic); those that did not are highlighted in bold. This table also shows the orientation (relative to infusion target), the bregma level and the connectivity of each region of interest. Tau propagation occurred in regions with direct connections (highlighted in bold) with the hippocampal formation and not to those without such connections (highlighted in italic); relative proximity to the site of infusion (bregma -2.50) did not influence tau propagation

$B E$ brain extract, $A / P$ anterior/posterior, Sup supplementary

connected to the hippocampal formation, it is in close proximity to the dentate gyrus, layer CA3 and the lower infusion coordinate (Supplementary Fig. 1). Despite this close proximity, the LGN did not show evidence of tau propagation (Fig. 3j); however, it is prone to ${ }^{\mathrm{P} 301 \mathrm{~S}} \mathrm{BE}$-associated tau pathology when targeted by a more ventral infusion coordinate (dural surface: DV -2.80 ; data not shown). Other brain regions, such as the ventrolateral thalamic nucleus (VLthalamus; bregma -1.00), the striatum (bregma 1.00) and the globus pallidus (bregma -1.00), are not directly connected to the hippocampal formation and did not show evidence of tau propagation in ${ }^{\mathrm{P} 301 \mathrm{~S}} \mathrm{BE}$ mice. Analysis of tau propagation in the cerebral cortex can be found in supplementary materials.

\section{Relationship between connectivity and tau propagation}

It is of note that all brain regions (Table 3 ) that showed evidence of tau propagation are directly connected to the hippocampus (dentate gyrus, CA3 or CA1) and/or the wider hippocampal formation (including subiculum and parahippocampal region). Those that did not show evidence of tau propagation, irrespective of relative proximity, are not directly linked to the hippocampal formation. Tau spread to distal sites (e.g. mIMN) occurred relatively quickly, in both a retrograde (suMN) and anterograde (mlMN) fashion.

Tau pathology in white matter that connects the hippocampal formation to regions showing evidence of tau propagation

The hippocampal formation is associated with three major white matter tracts: the angular bundle, the hippocampal commissure and the fimbria-fornix [2]. The hippocampal commissure represents the connection between right and left hippocampi, while the fimbria-fornix connects the hippocampal formation with the basal forebrain (e.g. LSN) and hypothalamic (e.g. mIMN and suMN) regions. As these regions (along with the hippocampal formation) 
Fig. 3 Propagation of tau pathology to brain regions distal to the infusion site. The density of PG-5-positive neurons or the area occupied by PG-5-positive immunoreactivity (tau burden; \% area) was quantified in discrete brain regions distal to the site of infusion. This analysis was conducted in ${ }^{\mathrm{P} 301 \mathrm{~S}} \mathrm{BE}$ (red) and PBS (green) mice 2.5 months post-infusion, both on the infused (solid bars) and the contralateral (checked bars) sides. Statistical analysis identified regions with significantly more tau pathology in ${ }^{\mathrm{P} 301 \mathrm{~S}} \mathrm{BE}$ mice than in PBS controls. The results of this analysis are summarised in Table 3. Tau propagation was detected in numerous distal sites $(\mathbf{a}-\mathbf{i})$, all of which have strong synaptic connections with the hippocampal formation. Regions not directly connected to the hippocampal formation (j-l) showed no evidence of tau propagation. Representative images showing PG-5 staining in retrosplenial cortex (m), lateral septal nucleus (n) and medial/lateral mammillary nucleus (o) serve to illustrate the severity and distribution of tau pathology in ${ }^{\mathrm{P} 301 \mathrm{~S}} \mathrm{BE}$ and PBS mice. RSC retrosplenial cortex, $m I M N$ medial/lateral mammillary nucleus, $s u M N$ supramammillary nucleus, $L S N$ lateral septal nucleus, ADthalamus anterodorsal thalamus, AVthalamus anteroventral thalamus, $L G N$ lateral geniculate nucleus, $B E$ brain extract, $P B S$ phosphate-buffered saline. Scale bars: $150 \mu \mathrm{m}$
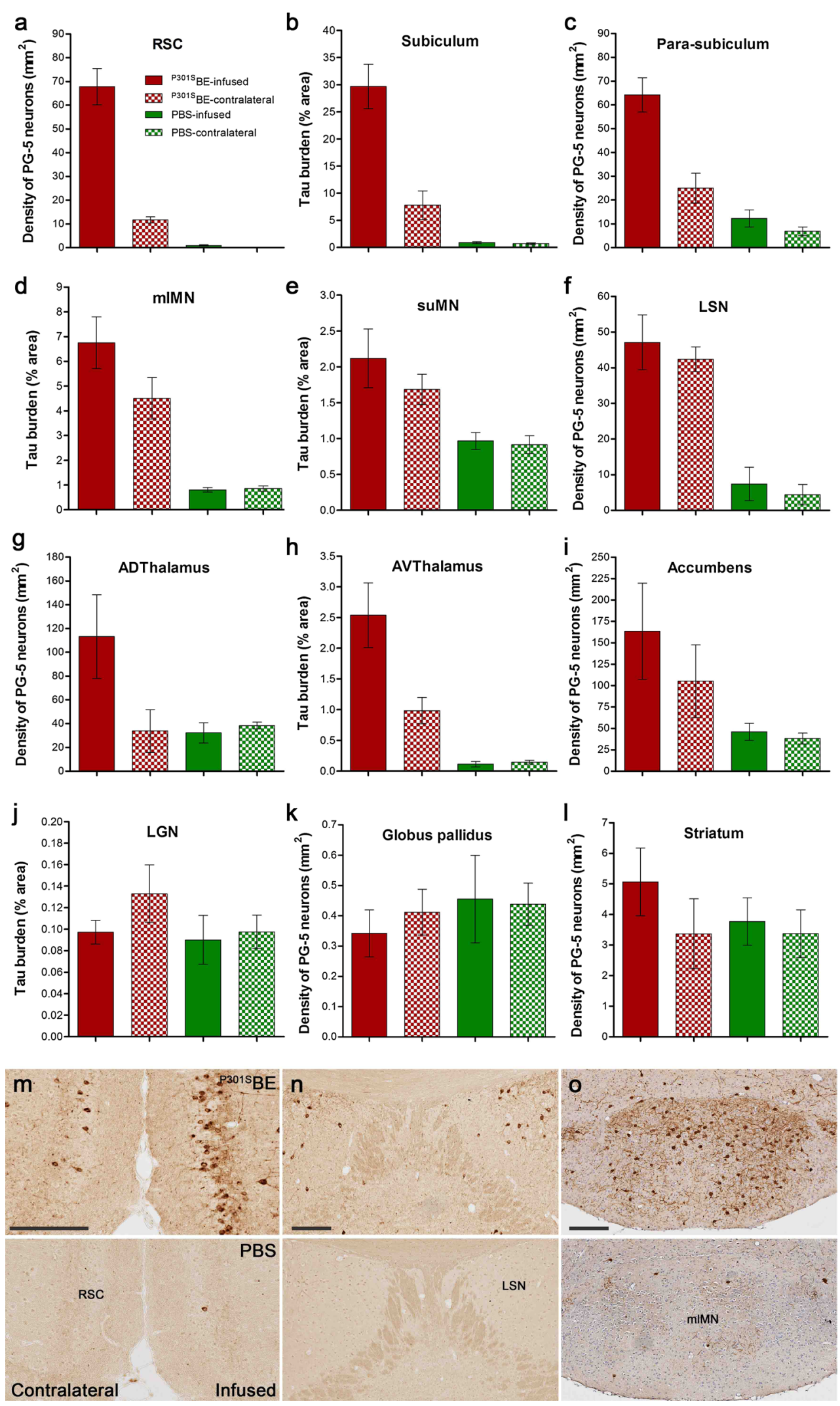

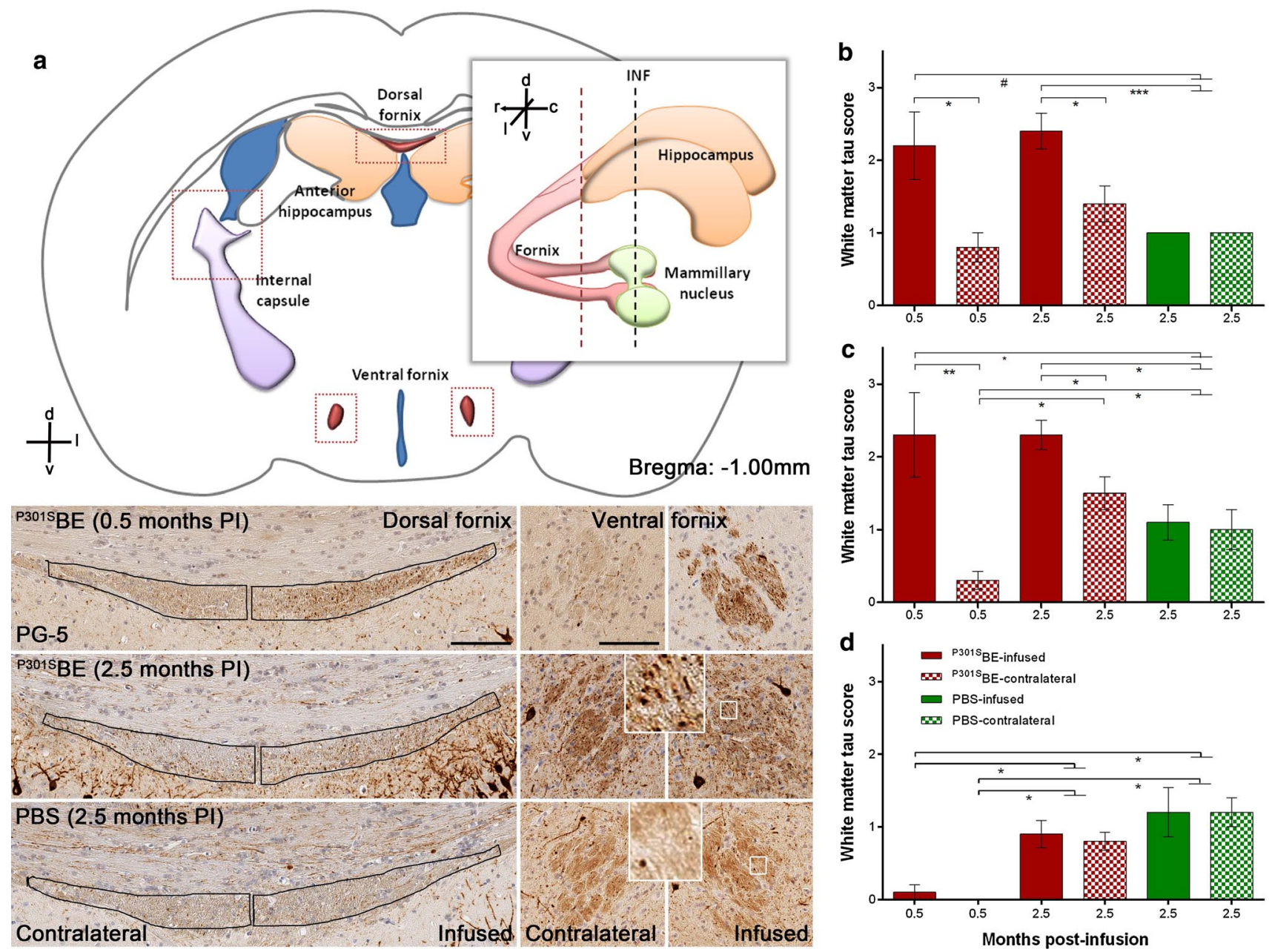

Fig. $4{ }^{\mathrm{P} 301 \mathrm{~S}}$ BE-induced tau pathology in white matter tracts. a The fornix (red) is a white matter tract that projects from the hippocampal formation (orange) to the basal forebrain structures, including the mammillary nucleus (green), both of which contain ${ }^{\mathrm{P} 301 \mathrm{~S}} \mathrm{BE}$-induced tau pathology (Table 3; Fig. 3). Representative images of the dorsal and ventral aspects of the fornix (bregma -1.00 ; corresponding to the red dotted line) showed PG-5-positive dot-like structures (insets) that were more numerous in ${ }^{\mathrm{P} 301 \mathrm{~S}} \mathrm{BE}$ mice than in controls. The morphology and location of these dot-like structures are compatible with tau aggregates in neuronal axons (cut in cross section; inset). Semiquantitative scoring in the dorsal (b) and ventral fornix (c) confirmed that tau pathology was more severe $(P<0.05)$ on the infused side of ${ }^{\mathrm{P} 301 \mathrm{~S}} \mathrm{BE}$ mice at 2 weeks $(0.5$ months $)$ and 2.5 months post-infusion when compared with controls. On the contralateral side, there was an increase in tau pathology between 2 weeks and 2.5 months in ${ }^{\mathrm{P} 301 \mathrm{~S}} \mathrm{BE}$ mice (significant for the ventral fornix). At both 2 weeks and 2.5 months, tau pathology was significantly more severe on the infused side of ${ }^{\mathrm{P} 301 \mathrm{~S}} \mathrm{BE}$ mice compared to the contralateral side. A similar analysis of the internal capsule (d) did not identify any ${ }^{\mathrm{P} 301 \mathrm{~S}}$ BE-induced changes in tau pathology, although there was a mild but significant increase between 2 weeks and 2.5 months postinfusion. Statistics: Groupwise comparison, Kruskal-Wallis test with Dunn's post hoc test; Pairwise comparison, Mann-Whitney test; ***, $P<0.001$; **, $P<0.01$; * $P<0.05$; \#, $P>0.05$ and $\leq 0.08$ (trend). $d$ dorsal, $l$ lateral, $v$ ventral, $r$ rostal, $c$ caudal, INF relative coronal level of the infusion site, $B E$ brain extract, $P B S$, phosphate-buffered saline. Scale bars: $100 \mu \mathrm{m}$

relatively infrequent in age-matched controls. They were absent at 1 day post-infusion (data not shown) and were numerous at 2 weeks, when they were largely confined to the infused side of the dorsal and ventral fornix (Fig. 4). Semi-quantitative scoring of PG-5-positive tau pathology confirmed these qualitative observations (Fig. 4b, c). Additionally, the severity of fornix tau pathology on the contralateral side of ${ }^{\mathrm{P} 301 \mathrm{~S}} \mathrm{BE}$ mice showed an increase between 2 weeks and 2.5 months, whereas it remained relatively the infused half compared to the contralateral half and were 
unchanged on the infused side. In contrast, the internal capsule was unaffected 2 weeks post-infusion and showed a mild, but significant increase in tau pathology at 2.5 months for both ${ }^{\mathrm{P} 301 \mathrm{~S}} \mathrm{BE}$ mice and control mice, with no difference between the infused and contralateral sides (Fig. 4d). The morphology and location of these 'dot-like' structures is consistent with tau aggregates in neuronal axons (observed in cross-section) [32]. Axonal localisation of these white matter tau aggregates in ${ }^{\mathrm{P} 301 \mathrm{~S}} \mathrm{BE}$ mice was more readily visible in the ventral hippocampal commissure (observed in longitudinal section) where tau pathology had a similar severity to that observed in the fornix (Supplementary Fig. 5). These findings suggest that the fornix progressively develops axonal tau pathology in response to the unilateral infusion of ${ }^{\mathrm{P} 301 \mathrm{~S}} \mathrm{BE}$ into the hippocampus, consistent with tau propagation, whereas the internal capsule does not.

No overt neurodegeneration associated with ${ }^{\mathrm{P} 301 \mathrm{~S}} \mathrm{BE}$-induced hippocampal tau pathology

Despite the severity of tau pathology in ${ }^{\mathrm{P} 301 \mathrm{~S}} \mathrm{BE}$-infused mice, there was no obvious nerve cell loss. A small subset of mice showed acute neuronal loss in the dentate gyrus and layer CA1 (Fig. 2a), but this was not specific to any of the treatment groups and was observed even 1 day post-infusion (data not shown), suggesting that it was unrelated to the accumulation of tau pathology and more likely associated with the mechanical nature of the infusion procedure. Analysis of Iba-1 staining for microgliosis was consistent with this notion (Supplementary materials). This analysis also identified a subtle increase in microgliosis in the oldest ${ }^{\text {P301S }}$ BE-infused mice (Supplementary Fig. 6) which might be indicative of an early inflammatory response to neurodegeneration.

\section{Discussion}

We adapted the human mutant P301S tau transgenic mouse line to develop a novel in vivo model of tau propagation, termed P301S-TP. In our model, ${ }^{\mathrm{P} 301 \mathrm{~S}} \mathrm{BE}$-induced tau pathology formed first in the hippocampus on the infused side, from where it spread to the contralateral hippocampus and to distal nuclei that are synaptically connected to the hippocampal formation. White matter tracts connecting the hippocampal formation to these distal nuclei also showed evidence of ${ }^{\mathrm{P} 301 \mathrm{~S}} \mathrm{BE}$-induced tau pathology.

P301S-TP as a rapid and robust model of tau propagation

The lack of significant tau pathology in the hippocampus of P301S tau mice infused with PBS or ${ }^{\mathrm{WT}} \mathrm{BE}$ indicates that this region is relatively spared at 4.5 months of age and that the induction and robust accumulation of tau pathology in P301S-TP mice is attributable to the infusion of ${ }^{\mathrm{P} 301 \mathrm{~S}} \mathrm{BE}$. The absence of tau pathology at 1 day shows that inclusions were not simply transferred from symptomatic to asymptomatic mice, but rather that the resulting intraneuronal deposits accumulated over time. Mild tissue disruption and associated microgliosis were observed 1 day postinfusion in both ${ }^{\mathrm{P} 301 \mathrm{~S}} \mathrm{BE}$ - and PBS-infused mice resulting from the needle tracks; however, this had no obvious effect on the induction of tau inclusions, since no differences were observed between infused and contralateral sides. It is unlike a recent study, which reported the rapid accumulation of oligomeric and phosphorylated tau in response to traumatic brain injury [24].

Temporal analysis of the hippocampus showed that the induction, maturation and spread of ${ }^{\mathrm{P} 301 \mathrm{~S}} \mathrm{BE}$-associated tau pathology occurred in a stereotypical and time-dependent manner resembling that in human brain, which underlies Braaks' NFT staging scheme [5]. We saw induction of hippocampal tau pathology within 2 weeks post-infusion, its contralateral spread within 1 month and a predominance of NFTs after 1 month, allowing us to effectively stage the progression of tau pathology in this novel mouse model. A transition from hyperphosphorylated, silver-negative to hyperphosphorylated, silver-positive tau inclusions in the P301S-TP model is also similar to the evolution of NFTs described in $\mathrm{AD}[3,4]$. Braak and colleagues described five groups based on hyperphosphorylated tau (antibody AT8) and Gallyas silver staining: (1) pre-tangles (only AT8 positive); (2) beginning of NFTs (AT8- and weakly Gallyas positive); (3) 'classic' NFTs (AT8- and Gallyas positive); (4) early ghost tangle (weakly AT8- and Gallyas positive); (5) extracellular 'ghost tangle' (weakly Gallyas positive) [4]. The majority of inclusions in the hippocampus of P301S-TP mice at 2 weeks post-infusion were pre-tangles and at 2.5 months NFTs. Extracellular tau inclusions were not observed, consistent with the lack of overt nerve cell loss. Similarly, only a small subset of PG-5-positive inclusions was thioflavin-S- or TG3 positive, which are markers of mature NFTs [26]. It is possible that further ageing of P301S-TP might increase the frequency of mature NFTs and result in neuronal loss; however, since P301S tau mice develop a severe paraparesis by 5-5.5 months of age [1], we did not age the mice beyond 4.5 months.

The first in vivo model of tau propagation showed a similar induction of tau pathology. Clavaguera et al. [9] used an identical ${ }^{\mathrm{P} 301 \mathrm{~S}} \mathrm{BE}$ preparation composed of hyperphosphorylated, filamentous tau and infused it into the ALZ17 line that overexpresses wild-type $4 \mathrm{R}$ human tau. Immunodepletion of tau from ${ }^{\mathrm{P} 301 \mathrm{~S}} \mathrm{BE}$ inhibited the induction and accumulation of pathology, indicating that its presence was essential for propagation. They also showed that overexpression of wildtype human tau was necessary for significant accumulation 
of pathology and that it formed the ${ }^{\mathrm{P} 301 \mathrm{~S}} \mathrm{BE}$-induced tau inclusions [9]. In the present study we observed a more rapid and robust induction of tau pathology than when ALZ17 was used as the recipient mouse line [9]. The ALZ17 model of propagation showed nerve cell body inclusions after 15 months and only modest contralateral spread even after 18 months [9]. Our P301S-TP model, using an identical infusion methodology (i.e. infusion preparation, coordinates, volume and flow rate), developed tau inclusions as early as 2 weeks post-infusion and showed contralateral hippocampal spread after 1 month. As the experimental methodologies were matched, the difference between models is likely to be related to the overexpression of human mutant P301S tau, which is more aggregation prone than WT human tau [21]. The concentration of total tau protein in ${ }^{\mathrm{P} 301 \mathrm{~S}} \mathrm{BE}$, which was threefold higher than ${ }^{\mathrm{WT}} \mathrm{BE}$ (supplementary materials), is unlikely to be the critical factor for initiating tau propagation in our model as it is also seen (to a lesser degree) when ${ }^{\mathrm{P} 301 \mathrm{~S}} \mathrm{BE}$ is diluted tenfold (data not shown). Endogenously expressed murine tau has been shown to accumulate in propagation-associated inclusions $[8,9,12,26]$ and is likely to be incorporated into inclusions formed in our P301STP model, although this was not tested. Collectively, these results are consistent with in vitro studies showing that synthetic tau fibrils recruited and converted endogenous soluble tau species into fibrillar aggregates [17, 23, 27, 49]. These features are also characteristic of other amyloidogenic proteins, such as $\alpha$-synuclein, amyloid $\beta$ and prion protein [19, 43]. Although the characteristics of tau that enabled seeding and templating are still unknown, it has been shown that tau extracts from human tauopathies $[8,30]$ and synthetic tau fibrils can induce tau pathology in vivo [10, 26].

Tau propagation is related to synaptic connectivity, not spatial proximity

Other models of tau propagation used either a tau infusion paradigm similar to the one used here $[9,26,30]$ or the region-specific expression of human mutant tau [12, 33]. Both approaches have suggested that tau pathology can spread to distal brain regions that are synaptically connected to the area where tau pathology was first induced. A potential weakness of the region-specific expression models is the possibility of low human mutant tau expression in the synaptically connected regions [42]. A drawback of the infusion models is the possibility of aggregated tau diffusing from the sites of infusion, forming a proximaldistal gradient and inducing pathology only in regions with a high basal expression of tau. Moreover, the connectivity of specific nuclei with and without propagation-associated pathology was not always examined or emphasised $[9,26]$.

Analysis of tau propagation in over 20 nuclei located anteriorly, posteriorly, ventrally or dorsally to the site of infusion provided conclusive evidence that tau pathology spread only to regions that were synaptically connected to the site of infusion (Fig. 5). Nuclei that are not directly connected to the hippocampal formation did not show evidence of tau propagation. The lack of tau propagation to the LGN, a nucleus not connected but in close proximity to the hippocampus, discounts the possibility of tau seeds simply diffusing from the infusion site to distal nuclei. The LGN forms tau inclusions when infused with ${ }^{\mathrm{P} 301 \mathrm{~S}} \mathrm{BE}$ (data not shown). In most nuclei showing evidence of ${ }^{\mathrm{P} 301 \mathrm{~S}} \mathrm{BE}$-induced tau propagation, the infused side was more severely affected than the contralateral side, both of which were affected more than either side of ${ }^{\mathrm{WT}} \mathrm{BE}$ - or PBS- infused mice. The LSN displayed a different pattern of pathology with the infused and contralateral sides showing equally severe ${ }^{\mathrm{P} 301 \mathrm{~S}} \mathrm{BE}$-induced pathology. This bilateral spread of tau pathology is likely to be a reflection of the strong bilateral and unidirectional inputs from one side of the hippocampus to both sides of the LSN [2]. The correlation between the pattern of spread and the synaptic connectivity of the LSN lends further support to a mechanism of spread related to synaptic connectivity and not proximity. The lack of propagation-related tau pathology in the dorsolateral cortex, which is close in proximity to the dorsal infusion coordinate and contains the primary visual cortex (Fig. 5), suggests that the hippocampus is the major mediator of tau spread in this model.

A model of tau propagation, which used microfluidic chambers to separate somatodendritic and axonal compartments, has shown that aggregated tau can be internalised by both compartments and transported along the axon in either the anterograde or the retrograde direction [49]. Most nuclei showing evidence of tau propagation in the present study were hippocampal efferents and therefore reflected an anterograde axonal spread. For example, the mIMN receives strong, unidirectional and bilateral efferent connections from the hippocampal formation [2] and shows evidence of bilateral ${ }^{\mathrm{P} 301 \mathrm{~S}} \mathrm{BE}$-induced tau pathology. The hippocampal region receives afferent axons from neurons in the suMN, which also shows evidence of tau propagation [2]. Consistent with the in vitro model [49], this is likely to be an in vivo example of retrograde spread, where tau aggregates are internalised by the axonal terminals of suMN neurons and transported along their axons in retrograde fashion to seed more tau aggregation in the distally located somatodendritic compartment.

The concept of anterograde and retrograde transport of assembled tau was further supported by the identification of tau aggregates in the fornix of P301S-TP mice, most likely within axons, since tau accumulation in oligodendroglia was not observed. This stands in marked contrast to the ALZ17 line, where numerous coiled bodies were 


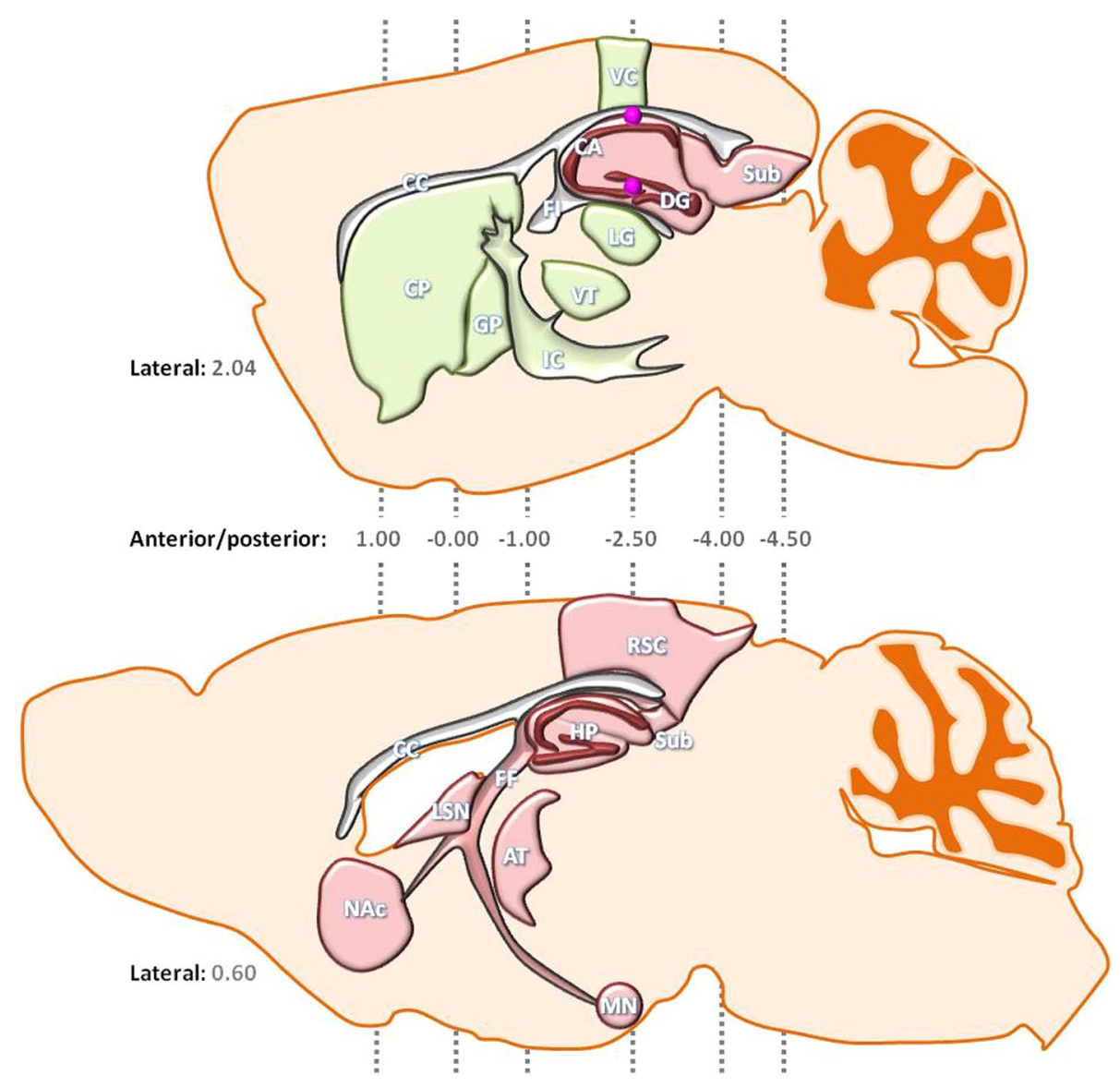

Fig. 5 Tau propagation is related to connectivity, and not proximity. Schematic of the mouse brain at two sagittal levels (lateral: 2.04 and $0.60)$ shows the structural relationship between nuclei with (red) and without (green) evidence of tau propagation. The former are synaptically connected to neurons in the hippocampus (dark red), the target of the ${ }^{\mathrm{P} 301 \mathrm{~S}} \mathrm{BE}$ infusion (purple dots). Nuclei located anteriorly and ventrally are connected to the hippocampus through the fimbriafornix (FF), which also showed evidence of tau propagation. The lateral geniculate nucleus (LG), which was proximal to the site of infusion, did not exhibit propagation and was not synaptically connected to the hippocampus, nor are nuclei associated with the striatonigral system that are interconnected through the internal capsule. This dis-

present following the infusion of either ${ }^{\mathrm{P} 301 \mathrm{~S}} \mathrm{BE}$ or brain extracts from human tauopathies [8,9]; the reason for this difference remains to be established. The relatively long distance $(\sim 5 \mathrm{~mm})$ from the infusion site to the mammillary nucleus via the fornix and the normally tightly regulated movement of proteins along axons suggest that the transport of aggregated tau species was probably an active process. If the purpose of this active transport is to neutralise abnormal tau species in the somatodendrictic compartment, this raises the question why tau would be anterogradely transported. The abnormal conformation/ post-translational modification of tau could be a factor leading to its apparent mislocalisation and ability/availability to propagate from cell to cell. tribution suggests that tau spreads to regions synaptically connected to the infusion site and not necessarily to regions in close proximity. Only specific nuclei were analysed for evidence of tau propagation (Table 3) and the sagittal levels were selected for illustrative purposes only. Grey dotted lines indicate anterior/posterior bregma levels in which nuclei were sampled (Supplementary Fig. 1). $A T$ anterior thalamus, $C A, C A 1-3, C C$ corpus callosum, $C P$ caudate/putamen, $D G$ dentate gyrus, $F F$ fimbria/fornix, $F I$ fimbria, $G P$ globus pallidus, $H P$ hippocampus, $I C$ internal capsule, $L G$ lateral geniculate nucleus, $L S N$ lateral septal nucleus, $M N$ mammillary nucleus, $N A c$ nucleus accumbens, $R S C$ retrosplenial cortex, $S u b$ subiculum, $V C$ visual cortex, $V T$ ventral thalamus

Is the P301S-TP model relevant to human diseases?

It has long been thought that abnormal tau accumulates in a cell-autonomous manner and that its distribution reflects the greater vulnerability of some neuronal populations, possibly related to variations in tau expression. Although this may be true in part, the results of this study and other in vivo models of tau propagation $[9,12,26,30,33]$ highlight the ability of tau to affect whole neuronal networks as opposed to isolated neurons/nuclei. There is growing evidence that network-dependent or 'trans-synaptic' spread of tau pathology also occurs in human tauopathies. Firstly, Braaks' staging suggests that tau pathology occurs early in the transentorhinal region, from where it spreads to the 
hippocampus and the neocortex [5]. The perforant pathway is likely to facilitate this apparent spread, as it originates from the entorhinal cortex and is the major source of input for the hippocampal formation [2], a view supported by the expression models of tau propagation $[12,33]$. Secondly, the nuclei that are affected in human tauopathies do not appear to be random, but form part of large-scale neuronal networks. Network-dependent spread could help to explain the selective vulnerability of the limbic system in $\mathrm{AD}$ or the severe pallido-nigro-luysial involvement in PSP and CBD [13, 37, 41]. Finally, network-dependent spread could account for heterogeneity within a distinct diagnostic tauopathy. There is considerable homogeneity that enables the identification of tauopathies (e.g. morphology of tau inclusions), but there is also a degree of heterogeneity unrelated to disease severity, which has led to the definition of clinicopathological variants of disease [14, 28, 34]. Thus, posterior cortical atrophy is an atypical variant of $\mathrm{AD}$ characterised by severe NFT pathology in the occipitoparietal cortex, with relative sparing of the hippocampus [46]. Network-dependent spread could explain this, if the initial tau aggregation was a stochastic event that was followed by deterministic spreading dependent on network connectivity. This concept could be put to the test in the P301S-TP model using different infusion coordinates.

Together with the present work, in vivo and human studies highlight the potential importance of the white matter in facilitating the spread of tau pathology. It is of note that the white matter is severely affected in PSP and CBD [13], and has been previously implicated in the spread of tau pathology in $\mathrm{AD}$ and other human tauopathies [29, 37, 41]. Manipulation of these discrete functional connections in the P301S-TP model through white matter transections, grey matter lesions or pharmacological manipulation is likely to shed light on the mechanism(s) of network spread, i.e. it could be used to investigate if spread is truly "transsynaptic" $[12,33]$.

\section{Downstream consequences of tau propagation}

In contrast to the parent line, which develops nerve cell loss in the anterior horn of the spinal cord and other parts of the central nervous system, P301S-TP mice did not develop frank neurodegeneration, consistent with other infusion models of tau propagation [9, 26, 30]. With tau pathology being minimal in the hippocampus (at 4.5 months) and severe in the spinal cord of the parent line [1], the disparity in neurodegeneration may to be related to the presumed lower expression of human P301S tau transcripts in the hippocampus. Thus, higher transgene expression in the spinal cord may be required to drive neurodegeneration, whereas a combination of lower hippocampal expression and the transient nature of the infusion procedure may be sufficient for inclusion formation, but not toxicity. This is reminiscent of the inhibition of neurodegeneration after transgene suppression in conditional models of tauopathy, despite the continued formation of NFTs [39]. These observations imply that propagation is necessary, but not sufficient for neurodegeneration and that the molecular species responsible for propagation and toxicity may be different $[8,11]$; the relevant molecular tau species remain to be identified.

Another possibility is that hippocampal neuronal loss may eventually develop upon further ageing of P301S$\mathrm{TP}$ mice, as significant neuronal loss in the parent line was reported at 6 months. The oldest P301S-TP mice (4.5 months) showed subtle evidence of increased microgliosis in layer CA3, a region affected early and severely by tau pathology and devoid of infusion-related damage. Increased microgliosis could be indicative of a neurodegenerative process, since microglia monitor the brain microenvironment and respond to tissue injury and degeneration $[35,45]$.

\section{Conclusion}

The P301S-TP model shows rapid and robust tau pathology, which progresses in a stereotypical and time-dependent manner similar to that observed in human tauopathies. These characteristics make it a suitable and flexible assay system for the development and testing of tau-based treatment strategies, with the ultimate aim of finding intervention and prevention strategies for human tauopathies. The characterisation of pathology over time should help tailor the present model to reflect pre-pathological (1 day2 weeks post-infusion) mild/early ( 2 weeks- 1 month post-infusion) or severe/late (1-2.5 months post-infusion) stages of tauopathy, as it is likely that some therapeutic strategies will be more effective at particular stages of disease. The model will also help us to understand the mechanisms underlying network-dependent spread and provide answers to fundamental questions regarding the tau species responsible and the molecular mechanism(s) of tau propagation.

Acknowledgments The authors would like to thank Peter Davies (Albert Einstein College of Medicine, NY) for supplying tau-specific antibodies and other members of the ND-DHT (Neurodegenerative Diseases Drug Hunting Team) for helpful comments and suggestions. MT is supported by the Swiss National Foundation (310030_135214) and the VELUX Foundation. IL and MG are supported by U.K.'s Medical Research Council (U105184291). Dedicated to JNC.

Conflict of interest Zeshan Ahmed, Jane Cooper, Tracey K. Murray, Katya Garn, Mark A, Ward, Annalisa Cavallini, Samuel Jackson, Suchira Bose, Michael L. Hutton and Michael J. O’Neill are employees of Eli Lilly. 


\section{References}

1. Allen B, Ingram E, Takao M, Smith MJ, Jakes R, Virdee K, Yoshida H, Holzer M, Craxton M, Emson PC et al (2002) Abundant tau filaments and nonapoptotic neurodegeneration in transgenic mice expressing human P301S tau protein. J Neurosci 22:9340-9351

2. Andersen P (2007) The hippocampus book. Oxford University Press, USA

3. Bancher C, Brunner C, Lassmann H, Budka H, Jellinger K, Wiche G, Seitelberger F, Grundke-Iqbal I, Iqbal K, Wisniewski HM (1989) Accumulation of abnormally phosphorylated tau precedes the formation of neurofibrillary tangles in Alzheimer's disease. Brain Res 477:90-99

4. Braak E, Braak H, Mandelkow EM (1994) A sequence of cytoskeleton changes related to the formation of neurofibrillary tangles and neuropil threads. Acta Neuropathol 87:554-567

5. Braak H, Braak E (1991) Neuropathological stageing of Alzheimer-related changes. Acta Neuropathol 82:239-259

6. Brion JP (2006) Immunological demonstration of tau protein in neurofibrillary tangles of Alzheimer's disease. J Alzheimers Dis 9:177-185

7. Chai X, Wu S, Murray TK, Kinley R, Cella CV, Sims H, Buckner N, Hanmer J, Davies P, O’Neill MJ et al (2011) Passive immunization with anti-Tau antibodies in two transgenic models: reduction of Tau pathology and delay of disease progression. J Biol Chem 286:34457-34467

8. Clavaguera F, Akatsu H, Fraser G, Crowther RA, Frank S, Hench J, Probst A, Winkler DT, Reichwald J, Staufenbiel M et al (2013) Brain homogenates from human tauopathies induce tau inclusions in mouse brain. Proc Natl Acad Sci USA 110:9535-9540

9. Clavaguera F, Bolmont T, Crowther RA, Abramowski D, Frank S, Probst A, Fraser G, Stalder AK, Beibel M, Staufenbiel M et al (2009) Transmission and spreading of tauopathy in transgenic mouse brain. Nat Cell Biol 11:909-913

10. Clavaguera F, Lavenir I, Falcon B, Frank S, Goedert M, Tolnay M (2013) "Prion-like" templated misfolding in tauopathies. Brain Pathol 23:342-349

11. Collinge J, Clarke AR (2007) A general model of prion strains and their pathogenicity. Science 318:930-936

12. de Calignon A, Polydoro M, Suarez-Calvet M, William C, Adamowicz DH, Kopeikina KJ, Pitstick R, Sahara N, Ashe KH, Carlson GA et al (2012) Propagation of tau pathology in a model of early Alzheimer's disease. Neuron 73:685-697

13. Dickson D, Weller RO (2011) Neurodegeneration: the molecular pathology of dementia and movement disorders, 2 edn. Wiley, New York

14. Dickson DW, Ahmed Z, Algom AA, Tsuboi Y, Josephs KA (2010) Neuropathology of variants of progressive supranuclear palsy. Curr Opin Neurol 23:394-400

15. Drechsel DN, Hyman AA, Cobb MH, Kirschner MW (1992) Modulation of the dynamic instability of tubulin assembly by the microtubule-associated protein tau. Mol Biol Cell 3:1141-1154

16. Frost B, Diamond MI (2010) Prion-like mechanisms in neurodegenerative diseases. Nat Rev Neurosci 11:155-159

17. Frost B, Jacks RL, Diamond MI (2009) Propagation of tau misfolding from the outside to the inside of a cell. J Biol Chem 284:12845-12852

18. Gallyas F (1971) Silver staining of Alzheimer's neurofibrillary changes by means of physical development. Acta Morphol Acad Sci Hung 19:1-8

19. Goedert M, Clavaguera F, Tolnay M (2010) The propagation of prion-like protein inclusions in neurodegenerative diseases. Trends Neurosci 33:317-325

20. Goedert M, Ghetti B (2007) Alois Alzheimer: his life and times. Brain Pathol 17:57-62
21. Goedert M, Jakes R, Crowther RA (1999) Effects of frontotemporal dementia FTDP-17 mutations on heparin-induced assembly of tau filaments. FEBS Lett 450:306-311

22. Grundke-Iqbal I, Iqbal K, Tung YC, Quinlan M, Wisniewski HM, Binder LI (1986) Abnormal phosphorylation of the microtubuleassociated protein tau (tau) in Alzheimer cytoskeletal pathology. Proc Natl Acad Sci USA 83:4913-4917

23. Guo JL, Lee VM (2011) Seeding of normal Tau by pathological Tau conformers drives pathogenesis of Alzheimer-like tangles. J Biol Chem 286:15317-15331

24. Hawkins BE, Krishnamurthy S, Castillo-Carranza DL, Sengupta U, Prough DS, Jackson GR, DeWitt DS, Kayed R (2013) Rapid accumulation of endogenous tau oligomers in a rat model of traumatic brain injury: possible link between traumatic brain injury and sporadic tauopathies. J Biol Chem 288:17042-17050

25. Hutton M, Lendon CL, Rizzu P, Baker M, Froelich S, Houlden $\mathrm{H}$, Pickering-Brown $\mathrm{S}$, Chakraverty $\mathrm{S}$, Isaacs $\mathrm{A}$, Grover $\mathrm{A}$ et al (1998) Association of missense and 5'-splice-site mutations in tau with the inherited dementia FTDP-17. Nature 393:702-705

26. Iba M, Guo JL, McBride JD, Zhang B, Trojanowski JQ, Lee VM (2013) Synthetic tau fibrils mediate transmission of neurofibrillary tangles in a transgenic mouse model of Alzheimer's-like tauopathy. J Neurosci 33:1024-1037

27. Kfoury N, Holmes BB, Jiang H, Holtzman DM, Diamond MI (2012) Trans-cellular propagation of Tau aggregation by fibrillar species. J Biol Chem 287:19440-19451

28. Kouri N, Murray ME, Hassan A, Rademakers R, Uitti RJ, Boeve BF, Graff-Radford NR, Wszolek ZK, Litvan I, Josephs KA et al (2011) Neuropathological features of corticobasal degeneration presenting as corticobasal syndrome or Richardson syndrome. Brain 134:3264-3275

29. Lace G, Savva GM, Forster G, de Silva R, Brayne C, Matthews FE, Barclay JJ, Dakin L, Ince PG, Wharton SB (2009) Hippocampal tau pathology is related to neuroanatomical connections: an ageing population-based study. Brain 132:1324-1334

30. Lasagna-Reeves CA, Castillo-Carranza DL, Sengupta U, Guerrero-Munoz MJ, Kiritoshi T, Neugebauer V, Jackson GR, Kayed $\mathrm{R}$ (2012) Alzheimer brain-derived tau oligomers propagate pathology from endogenous tau. Sci Rep 2:700

31. Lee VM, Goedert M, Trojanowski JQ (2001) Neurodegenerative tauopathies. Annu Rev Neurosci 24:1121-1159

32. Lin WL, Zehr C, Lewis J, Hutton M, Yen SH, Dickson DW (2005) Progressive white matter pathology in the spinal cord of transgenic mice expressing mutant (P301L) human tau. J Neurocytol 34:397-410

33. Liu L, Drouet V, Wu JW, Witter MP, Small SA, Clelland C, Duff K (2012) Trans-synaptic spread of tau pathology in vivo. PLoS ONE 7:e31302

34. Murray ME, Graff-Radford NR, Ross OA, Petersen RC, Duara R, Dickson DW (2011) Neuropathologically defined subtypes of Alzheimer's disease with distinct clinical characteristics: a retrospective study. Lancet Neurol 10:785-796

35. Nimmerjahn A, Kirchhoff F, Helmchen F (2005) Resting microglial cells are highly dynamic surveillants of brain parenchyma in vivo. Science 308:1314-1318

36. Orrison WW (2008) Atlas of brain function. Georg Thieme Verlag, Stuttgart

37. Pearson RC, Esiri MM, Hiorns RW, Wilcock GK, Powell TP (1985) Anatomical correlates of the distribution of the pathological changes in the neocortex in Alzheimer disease. Proc Natl Acad Sci USA 82:4531-4534

38. Poorkaj P, Bird TD, Wijsman E, Nemens E, Garruto RM, Anderson L, Andreadis A, Wiederholt WC, Raskind M, Schellenberg GD (1998) Tau is a candidate gene for chromosome 17 frontotemporal dementia. Ann Neurol 43:815-825 
39. Ramsden M, Kotilinek L, Forster C, Paulson J, McGowan E, SantaCruz K, Guimaraes A, Yue M, Lewis J, Carlson G et al (2005) Age-dependent neurofibrillary tangle formation, neuron loss, and memory impairment in a mouse model of human tauopathy (P301L). J Neurosci 25:10637-10647

40. Rudelli RD, Ambler MW, Wisniewski HM (1984) Morphology and distribution of Alzheimer neuritic (senile) and amyloid plaques in striatum and diencephalon. Acta Neuropathol 64:273-281

41. Saper CB, Wainer BH, German DC (1987) Axonal and transneuronal transport in the transmission of neurological disease: potential role in system degenerations, including Alzheimer's disease. Neuroscience 23:389-398

42. Soto C (2012) In vivo spreading of tau pathology. Neuron 73:621-623

43. Soto C (2012) Transmissible proteins: expanding the prion heresy. Cell 149:968-977

44. Spillantini MG, Murrell JR, Goedert M, Farlow MR, Klug A, Ghetti B (1998) Mutation in the tau gene in familial multiple system tauopathy with presenile dementia. Proc Natl Acad Sci USA 95:7737-7741

45. Streit WJ (2002) Microglia as neuroprotective, immunocompetent cells of the CNS. Glia 40:133-139

46. Tang-Wai DF, Graff-Radford NR, Boeve BF, Dickson DW, Parisi JE, Crook R, Caselli RJ, Knopman DS, Petersen RC (2004) Clinical, genetic, and neuropathologic characteristics of posterior cortical atrophy. Neurology 63:1168-1174

47. Wilcock GK, Esiri MM (1982) Plaques, tangles and dementia, a quantitative study. J Neurol Sci 56:343-356

48. Witman GB, Cleveland DW, Weingarten MD, Kirschner MW (1976) Tubulin requires tau for growth onto microtubule initiating sites. Proc Natl Acad Sci USA 73:4070-4074

49. Wu JW, Herman M, Liu L, Simoes S, Acker CM, Figueroa H, Steinberg JI, Margittai M, Kayed R, Zurzolo C et al (2013) Small misfolded Tau species are internalized via bulk endocytosis and anterogradely and retrogradely transported in neurons. J Biol Chem 288:1856-1870 